ci marquera une nouvelle progression considérable vers le but poursuivi : création d'une forme à croissance rapide de la Carpe à écailles de notre pays, adaptée à nos étangs moyens.

En récapitulant ces résultats, on ne peut s'empècher de penser que le distingué pisciculteur de Fismes n'a pu donner à ses travaux ni l'ampleur, ni les soins qu'il eût désiré leur réserver et que, si les considérations commerciales qui s imposaient à lui ne l'avaient entravé, il se fût, sans doute, rapproché plus vite encore du type précoce et de forme avantageuse qu'il recherche.

Les pisciculteurs français ne peuvent que former des vceux pour qu'aucun obstacle ne vienne arrêter M. Valbors dans l'achèvement de l'cuure si belle et si attachante, mais.si longue et si délicate, qu'il a eu le courage d'entreprendre.

\title{
LA MESURE DU pH DES EAUX D'ÉTANGS
}

\author{
Par M. G. GALLICE \\ Prosident de l'Lnion piseicole de France et de l'Lnion nationale \\ des Syndicats de l'Etang.
}

II. DE Bouville aura renḍu un grand service aux propriétaires d'étangs cn obtenant du Docteur W. Wunder l'article publié par le Bulletin de Mars dernier ( $\mathrm{I}$ ).

Nous savions tous que la plupart des étangs au sous-sol argileux étaient améliorés par des apports d'amendements calcaires.

MM. les Professeurs LÉger et Roule avaient insisté sur l'importance de cette pratique pour la production du plancton.

Faut-il rappeler également que, chaque fois qu'on enlève roo kilogrammes de Poisson d'un étang, on prélève-sur le fond les $8 \mathrm{k}$. 400 de carbonate de calcium et les $5 \mathbf{k}$. 600 de phosphate de calcium qui forment le squelette?

Ce que nous ignorions, c'est qu'un mode d'analyse très précis permet à tout exploitant tant soit peu soigneux de se rendre compte du degré d'acidité ou d'alcalinité de l'eau et, par suite, de régler le chaulage de façon à atteindre le rendement optimum.

Le Docteur Wunder fait une courte description du foliocolorimètre de Wulfr et montre comment, à l'aide de cet appareil, on peut, en quelques minutes, mesurer le degré de l'eau en pHl (puissance d'hydrogène).

L'eau est acide si le pH trouvé est compris entre o et 7 ; neutre pour pH $=7$; alcaline si le $\mathrm{pH}$ est compris entre $7, \mathrm{I}$ et $\mathrm{I} / 4$.

Pour montrer toute l'importance de cette analyse, nous allons donner quelques chiffres.

(1) Page 197 .

Article available at http://www.kmae-journal.org or http://dx.doi.org/10.1051/kmae:1930010 
M. le Professeur Roule a bien voulu, récemment, se déplacer avec son assistant, M. CARIÉ, pour analyser l'eau de trois de nos étangs. Deux de ces étangs sont en pleine forêt, sur sol argileux, et leur rendement est médiocre ; à la température de $r 9^{\circ} \mathrm{C}$. on a trouvé pour le $\mathrm{pH}$ les nombres $6, \mathrm{r}$ et 6,6 ; l'eau est donc franchement acide. Le troisième étang, bien meilleur, reçoit les eaux de terres cultivées et de prairies, son $\mathrm{pH}$ s'élève à 6,8 ; l'eau est presque neutre.

Quelques jours auparavant, j'avais eu l'occasion de faire l'analyse de l'eau d'étangs situés sur un terrain calcaire et réputés pour leur haut rendement ; le pH s'est élevé à 7,8 . L'eau est donc franchement alcaline.

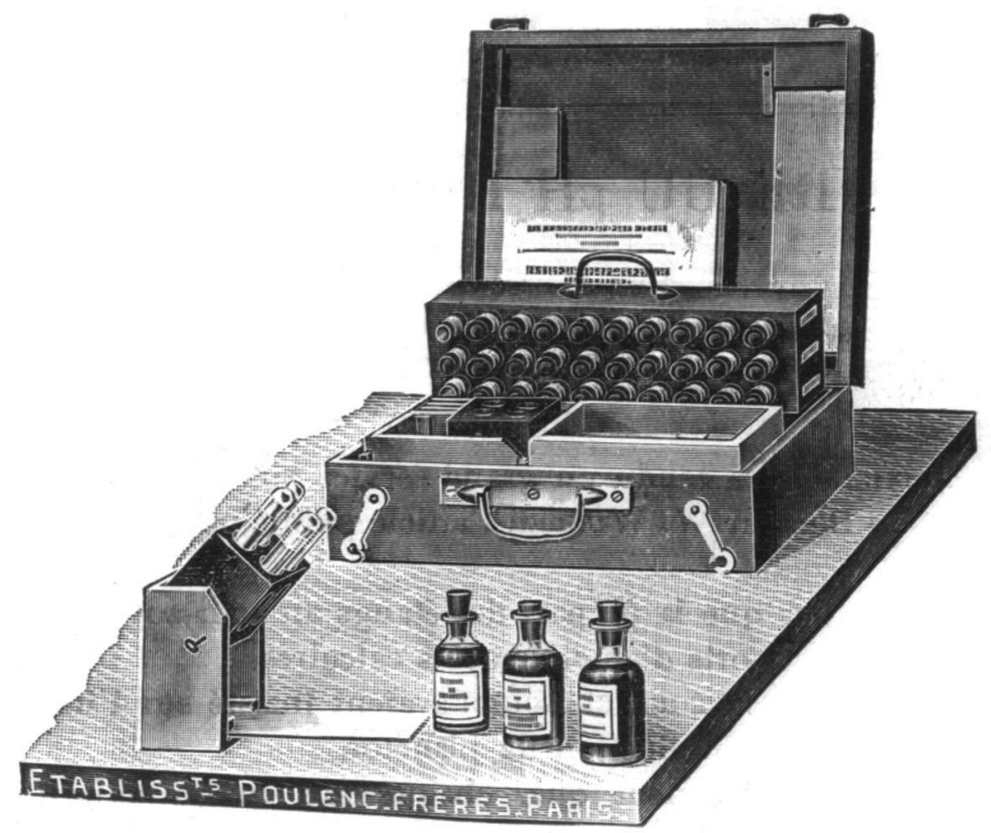

Fig. 10. - Nécessaire Bruk̇e pour la mesure du pH.

Le foliocolorimètre de WulfF n'est pas le seul appareil dont on puisse se servir.

Il existe, en France, un excellent nécessaire colorimétrique dú au Docteur Bruère, docteur ès sciences, pharmacien-colonel de l'armée ; c'est, d'ailleurs, ce nécessaire qu'emploie le Professeur Roule (r).

Le nécessaire BRuk̀re comprend trois échelles colorimétriques formées par des liquides colorés, inaltérables, enfermés dans des tubes scellés ; un bloc compensateur pour l'examen du liquide ; cinq flacons de réactifs, des tubes et des éprouvettes en verre neutre.

(1) Le nécessaire colorimétrique Bruk̀ne est construit par la Société des Usines Chimiques Rhone-Poulenc, r22, boulevard Saint-Germain. Cette firme, que nous venons de consulter, nous informe qu'elle vient de mettre au point un nouveau mécessaire ¿ 5 réactifs. - La manipulation dé ce nécessaire reste aussi simple, mais l'étendue de ses applications est plus considérable. - Son prix est de 380 francs. 
Un peu plus encombrant que le foliocolorimètre, cet appareil présente sur ce dernier deux avantages que je considère comme très importants :

$I^{\circ}$ Un premier essai, très rapide, consiste à verser une ou deux gouttes de réactif bleu dans un centimètre cube de l'eau à analyser ; le changement immédiat de teinte détermine sans ambiguïté quel réactif il convient de choisir pour l'analyse et, ipso facto, dans quelle échelle colorimétrique se trouvera la teinte de comparaison.

Avec le foliocolorimètre, pour les valeurs de $\mathrm{pH}$ voisines de 7 , il peut $\mathrm{y}$ avoir doute ou tâtonnement pour le choix des pellicules à employer.

$2^{\circ}$ L'eau à analyser est rarement incolore. Dans les étangs de bois, elle est presque toujours colorée en jaune ou en brun rougeâtre.

Le bloc compensateur du nécessaire Bruène permet de tenir compte de cette teinte initiale qui, sans lui, pourrait fausser le résultat de l'analyse.

Incliné à 45 degrés et éclairé par une plaque d'opaline blanche, ce bloc cst percé de quatre trous.

Dans les deux trous de gauche on met un tube d'eau distillée et l'eau qui a reçu le réactif ; dans les trous de droite, le test coloré et l'eau de l'étang. La teinte naturelle de l'eau figure donc des deux côtés : il y a "compensation ".

\section{TRAVAUX FRANÇAIS SUR LE $\mathrm{pH}$.}

A. LABre. - Les variations de la concentration en ions hydrogène dans les marais salants comme milieu biologique, - C. R. de l'Académie des Sciences, CLXXV, Paris, 1923.

La distribution des animaux des marais salants dans ses rapports avec la concentration en ions hydrogène, - Ibid.

Les zones critiques de l'adaptation au milieu, -- C. R. Acad. Sciences, CI.XXVI, I 923.

Ine conception nowvelle de l'adaptation : l'allélogénèsc, - Revue scientifique, LXII, Paris, 1923.

L'action biologique du $\mathrm{pH}$ ascendant de l'eau de mer et l'allélogénèse, - Revue générale des Sciences, XXXIV, Paris, r92\%.

Contribution à l'étude des milieux marins hyperalcalins, - Archives de zoologic expérimentale et générale. LXII, Paris, rgał.

Contribution à l'étude de l'allélogénèse : - le cycle biologique de Dunaliella, Archives d'anatomic microscopique, XXI, Paris, 1926.

Contribution à l'étude de l'allélngx́nc̀se : - essai d'une théorie des adaptations, Bulletin biologique de la France et de la Belgique, LX, Paris, I 926.

Contribution à l'étude de l'allélogénese' ; - l'histoire naturelle des Copépodes des marais salants du Croisic, - Archive de zoologie expérimentale, LXVI, Paris, rgá7.

R. Legexdrn. - Variations de la concenlration en ions-hydrogène de l'eau de mer littorale, - C. R. de l'Acarímie des Sciences, CLXXV, Paris, 1922.

Variation de concentration des ions-hydrogène de l'eau des mares supra littorales a Harpacticus fulvus, - C. R. de la Soriété de Biologie, LXXXIX, Paris, r $9^{23}$.

Variation de concentration en ions-hydrogène des fonds marins littoraux, - Ibid:, xC, 1924 .

La concentration en ions-hydrogìne de I'eau de mer : le $\mathrm{pH}, \ldots$ - Paris, Presses Universitaires, I $9: 5$.

Sogitte Rhone-Poulenc. - Nolice sur le nécessaire colorimétrique Bruère. 